\title{
Nuevos avances en relación con las prácticas económicas de los grupos pedemontanos de la cuenca del San Francisco (noroeste de Argentina, 800 a.C. -500 d.C.) a través de marcadores biomoleculares y microrrestos vegetales
}

\author{
Gabriela ORTIZ \\ CONICET - Facutad de Humanidades y Ciencias Sociales, Universidad Nacional de Jujuy (Argentina) \\ yolatordo@hotmail.com \\ Cecilia HeIT \\ Laboratorio de Análisis de Residuos y Trazas, Universidad Nacional de Jujuy (Argentina) \\ cheit@imagine.com.ar
}

Recibido: 23 de marzo de 2012

Aceptado: 24 de octubre de 2013

\begin{abstract}
RESUMEN
Se presentan los resultados de análisis de cromatografía gaseosa para la detección de marcadores biomoleculares (ácidos grasos) efectuados en fragmentos cerámicos asociados a la Tradición San Francisco (800 a.C.-500 d.C.). Estos análisis fueron complementados con estudios de almidones y fitolitos a efectos de determinar con mayor precisión las sustancias y recursos que estuvieron en contacto con las paredes de los contenedores cerámicos. Se discuten las implicaciones de los resultados a la luz de los modelos económicos tradicionalmente propuestos para las sociedades que ocuparon la región pedemontana del valle de San Francisco.
\end{abstract}

Palabras clave: Tradición San Francisco, región Yungas, análisis biomoleculares.

\section{New Advances in Relation to Economic Practices of San Francisco Valley Foothill Groups (Northwest Argentina, 800 BC-AD 500) Trough Biomolecular Markers and Vegetable Micro Remains}

\begin{abstract}
The results of gaseous chromatography analyses for bio-molecular (fat acids) markers carried out on pottery fragments associated to San Francisco Tradition (800 BC-AD 500) are presented. These analyses were complemented with starch and phytolite studies in order to determine more accurately substances and resources that were in contact with pottery container walls. Implications of the results are discussed in the light of economic models traditionally proposed for societies that occupied the foothill region of the San Francisco Valley.
\end{abstract}

Key words: San Francisco Tradition, Yungas region, biomolecular analysis.

Sumario: 1. Introducción: prácticas económicas de los grupos adscritos a la Tradición San Franscisco. 2. Análisis de marcadores biomoleculares y microrrestos vegetales. 3. Discusión. 4. Referencias bibliográficas.

\section{Introducción: prácticas económicas de los grupos adscritos a la Tradición San Francisco}

El valle de San Francisco se encuentra ubicado en la región pedemontana de la provincia de Jujuy, Argentina. Ambientalmente se caracteriza por su biodiversidad; la selva Tucumano-Oranense o Yungas de montaña enclavada en el dominio chaqueño, con su clímax en las laderas de 1.000 a 2.000 msnm -piso que recibe el mayor impac- 


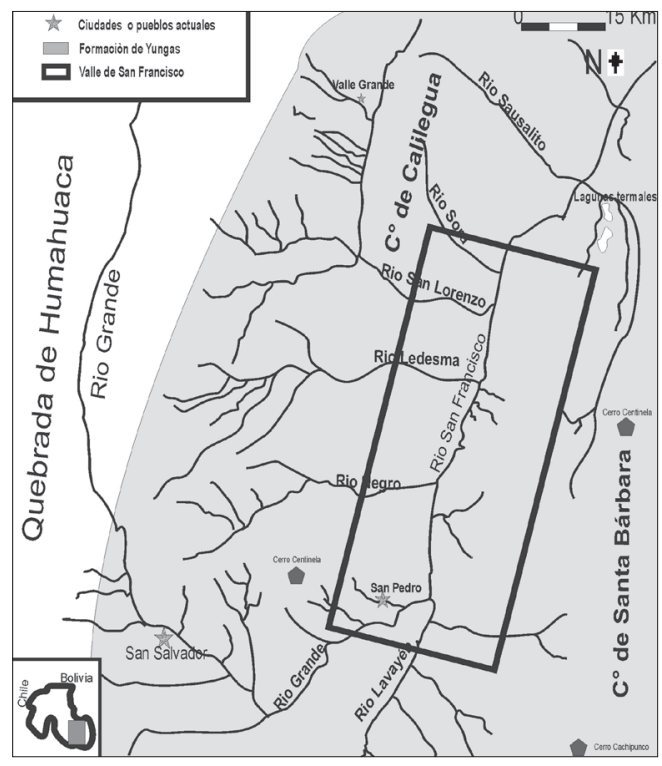

Figura 1: Región del valle del río San Francisco.

to de las lluvias orográficas-constituye la masa arbórea más densa de la Argentina, que incluye especies tales como el urundel (Loxopterygium grisebachii), el palo blanco (Calycophyllum multiflorum), y la tipa (Tipuana tipu), etc. Por encima de los $2.000 \mathrm{msnm}$ aparece el bosque de alisos (Alnus acuminata kunth) y queñoas (Polylepis tomente$l l a)$. Es también un enclave faunístico $\mathrm{y}$ un ámbito en el que se ha producido un significativo avance moderno de la agricultura a través de la implantación de cultivos de caña de azúcar, soja, porotos y otros múltiples frutos tropicales para el consumo local, algunos recientemente como el mango, las paltas y la papaya. Se practica la cría de ganado vacuno y caprino. La economía regional se ha estructurado a partir del desarrollo de la madera, la agricultura y los hidrocarburos.

El río San Francisco, que da nombre al valle, nace en la provincia de Jujuy, en la confluencia de los ríos Grande y Lavayén; desde allí sigue su curso de sur a norte, internándose en la provincia de Salta, donde desemboca en el río Bermejo (Figura 1). Este fértil valle estuvo ocupado por poblaciones prehispánicas desde aproximadamente el 800 a.C. Llama poderosamente la atención que, tras más de mil años de una ocupación continua, alrededor del 500 d.C. cesa la evidencia material de ocupación humana en la región, al menos hasta el siglo XV aproximadamente. Las poblaciones con que se encontraron los conquistadores europeos en el siglo XVI corresponden a grupos de raigambre chaqueña, de hábitos cazadores y recolectores. Esto es particularmente llamativo dado que se trata de una de las regiones más productivas de la provincia de Jujuy, incluso en mayor medida que regiones aledañas como la Quebrada de Humahuaca, que tuvo una ocupación continua a lo largo de toda la secuencia cultural del Noroeste de Argentina.

La llamada región del río San Francisco fue tempranamente explorada por lo miembros de la misión sueca a principios del siglo XX (Boman 1991 [1908]; Nordenskiöld 1993 [1903]), pero no fue sino a partir de la década de los años setenta que comenzaron las investigaciones arqueológicas sistemáticas (Dougherty 1975). Debido a sus particulares condiciones ambientales, se recuperaron escasos restos orgánicos en más de cien años de investigaciones; particularmente exiguos son aquellos atribuidos a especies vegetales. No obstante, dada la abundancia de los conjuntos materiales, especialmente la cerámica, se asumió que estos grupos fueron poblaciones agrícolas; hay que mencionar, asimismo, que el rango cronológico de la ocupación del valle por parte de estas poblaciones tempranas era coincidente con el desarrollo 
de las primeras aldeas «formativas» ${ }^{1}$ del noroeste de Argentina. Aunque algunos de los escasos restos arqueofaunísticos exhumados hasta la década de los años ochenta, así como la presencia de endocarpios quemados de plantas silvestres en un fogón, llevaron a considerar la posibilidad de una economía intensamente extractiva en donde la caza y la pesca jugaron un papel importante (Dougherty 1975), la supuesta presencia de agricultura fue el denominador más frecuente para caracterizar a estas poblaciones bajo el rótulo de «agroalfareros».

Con el avance de las investigaciones, particularmente en la última década, nuevas excavaciones en sitios con dataciones de comienzo de la era cristiana, así como la aplicación de técnicas orientadas a la recuperación de datos que aportaran información de primera mano sobre el uso de recursos en el pasado, han permitido empezar a

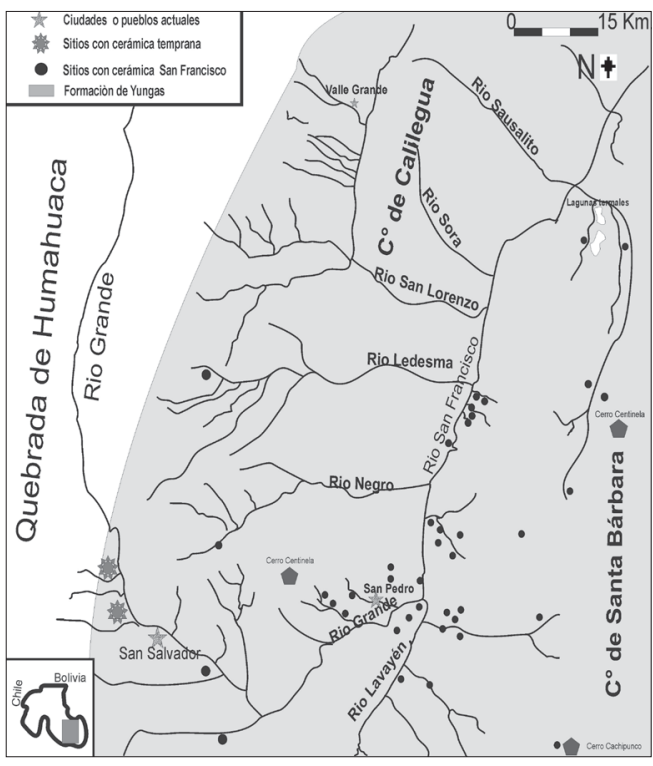

Figura 2: Sitios arqueológicos registrados en el valle del San Francisco (fuentes: Boman 1991 [1908]; Dougherty 1975; Dougherty et al. 2003; Fernández Distel 1994; Echenique y Kulemeyer 2003; Nordenskiöld 1993 [1903]; Ortiz 2007). pensar el estatus «formativo» de estas poblaciones (Ortiz 2007, 2011), al menos en lo relativo al contenido conceptual del término.

Los primeros estudios realizados sobre huesos humanos parecen apoyar la hipótesis de una dieta mixta (Seldes y Ortiz 2009). La ausencia de evidencia de estrés nutricional podría estar indicando una explotación óptima del ambiente con una estrategia económica basada en una dieta de amplio espectro. La inclusión de numerosas especies de animales de pequeño tamaño que soportan mayor presión de caza por sus altas tasas de renovación, el consumo de peces y el uso de algunos cultígenos serían claros indicios de un aumento en los costos de procesamiento y de la presión sobre espacios reducidos.

Un perfil polínico realizado en el sitio de Moralito (Lupo y Echenique 2001) muestra una variedad muy grande de taxones representados y una sobrerrepresentación de polen de Amaranthaceae y Chenopodiaceae. A su vez, muchos sitios exhiben unas estructuras de forma aproximadamente campanuliformes, conocidas en la literatura clásica arqueológica como «hornos en forma de campana». Cuando estos están presentes, aparecen en grupos y suponemos que fueron usados, al igual que los registrados en otros sitios del Noroeste Argentino (NOA) (ver especialmente Laguens 1993),

1 El término Formativo alude, en los modelos de desarrollo cultural del noroeste de Argentina, al período caracterizado por una vida aldeana sedentaria bien establecida, una economía de tipo agropecuario y la presencia de alfarería. 
para el procesamiento y almacenamiento de semillas de leguminosas. Si la función fue la de conservar y almacenar frutos a gran escala, sus dimensiones hablan de una capacidad de almacenamiento, en promedio, de $140 \mathrm{~kg}$.

En algunos de los sitios trabajados desde principios de siglo, se han reportado restos de ictiofauna, sin dejar de mencionar que de los 40 sitios arqueológicos registrados hasta el presente, 38 de ellos se encuentran a la vera de ríos o de paleocauces, lo que habla de la preferencia por asentarse en las proximidades de cursos fluviales (Figura 2). Los ambientes ribereños son especialmente ricos en términos de biodiversidad; en las selvas tropicales, estos ambientes ribereños con abundancia de recursos no habrían potenciado la emergencia de la producción vegetal a gran escala (Piperno y Pearsall 1998). Las poblaciones que explotan una variedad de recursos, entre los que se incluyen los peces de agua dulce, tienden a no depender exclusivamente de las plantas cultivadas, siendo estas un complemento en una dieta de amplio espectro.

Muchas sociedades prehispánicas que ocuparon ambientes de alto rendimiento encontraron soluciones provechosas, con economías sostenidas en el tiempo y viables en el ambiente local, que involucraban solamente un uso complementario de plantas domésticas (Smith 2001). Dado que la economía de estos grupos siempre fue asumida, pero no demostrada, los estudios conducidos en los últimos años se han centrado en la recuperación de evidencia confiable para discutir la importancia de determinados tipos de recursos en la economía de estas poblaciones. El manejo social del medio estaría reflejado en una baja movilidad, basada en la explotación intensiva de porciones reducidas de un ambiente de alto rendimiento. La abundancia de recursos, su procesamiento y almacenamiento a gran escala tienen a su vez una correspondencia altamente positiva con la abundancia y diversidad de los conjuntos materiales, especialmente los contenedores cerámicos.

En este contexto de investigación, los análisis de las sustancias que estuvieron contenidas en los recipientes de cerámica, como el que se presenta a continuación, añaden la potencialidad de determinar la posible existencia de plantas domésticas y su utilización asociada a determinados tipos de contenedores cerámicos. Esto a su vez nos capacita para empezar a discutir con bases sólidas la importancia relativa de especies silvestres y domésticas de plantas y animales en la economía de los tradicionalmente llamados grupos «formativos» de la región pedemontana de Jujuy.

\section{Análisis de marcadores biomoleculares y microrrestos vegetales}

Con objeto de comenzar a explorar la posible presencia de plantas domésticas en poder de estos grupos tempranos, se recurrió a distintos métodos para la detección de los contenidos de vasijas cerámicas. El objetivo fue complementar, a través de procedimientos analíticos diferentes, el estudio de aquellos recursos que pudieron estar involucrados en la preparación de alimentos, superando la barrera de los supuestos o consideraciones subjetivas con la obtención de pruebas directas. Ello permite, a su vez, ampliar el límite de las expectativas arqueológicas tradicionales, que están mayoritariamente enfocadas a considerar aquellos recursos exclusivamente asociados con prácticas agrícolas, limitando la visión en conjunto de todos los recursos 
que pudieron haber sido usados y las prácticas diversas involucradas en su manejo (Babot 2007).

Uno de los procedimientos consistió en el análisis de ácidos grasos de las superficies de tiestos cerámicos. Los otros dos apuntaron a la detección de fitolitos y almidones a partir de fragmentos cerámicos $\mathrm{y}$ sedimentos. Las muestras cerámicas seleccionadas corresponden a fragmentos pertenecientes a diferentes categorías de contenedores de acuerdo con su función primaria supuesta: vasijas de tipo cántaro, escudillas, botellas y ollas (Figura 3). Los fragmentos no fueron lavados y se enviaron para su análisis tal como se habían recuperado de la matriz sedimentaria de los sitios arqueológicos donde fueron desenterrados.

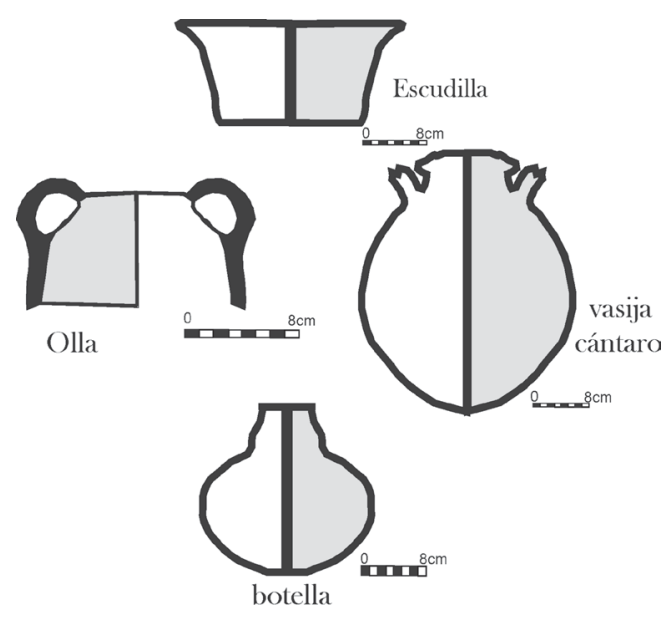

Figura 3: Perfiles reconstruidos de las principales formas de contenedores cerámicos.

\subsection{Análisis de ácidos grasos}

Los lípidos y ceras absorbidos por los recipientes cerámicos durante su utilización han demostrado preservarse por miles de años a pesar del ataque microbiano y del deterioro químico (Eerkens 2007). Muchas veces este tipo de datos proporciona la única evidencia confiable en lugares donde las condiciones de preservación para restos orgánicos son exiguas. La identificación de las materias primas usadas basada en los residuos de lípidos en recipientes cerámicos es inevitablemente complicada debido a los procesos de degradación que ocurren tanto durante el uso de las vasijas como durante el proceso posterior a su entierro en la matriz sedimentaria (Dudd y Evershed 1999). A pesar de esto, el estudio de marcadores biomoleculares se ha convertido en una poderosa herramienta que ha resultado ser, en numerosos casos, una forma provechosa de obtener información acerca de los recursos y sustancias que fueron procesadas en las vasijas cerámicas.

En esta oportunidad se seleccionaron 21 muestras de diferentes tiestos pertenecientes a las siguientes categorías: 3 fragmentos de escudillas; 5 fragmentos de botellas; 7 fragmentos de vasijas tipo cántaro y 6 fragmentos de ollas. Las muestras provienen de tres sitios arqueológicos (Pozo de la Chola, Aguas Negras y Fraile Pintado), todos adscritos a la Tradición San Francisco (Figura 4). Pozo de la Chola $(\mathrm{PdCH})$ y Aguas Negras (AN) cuentan con dataciones radiocarbónicas que ubican al primero a principios de la era cristiana y al segundo hacia el $500 \mathrm{~d}$. C. La muestra radiocarbónica de Fraile Pintado no puedo ser procesada debido a la mala preservación de la proteína ósea. 


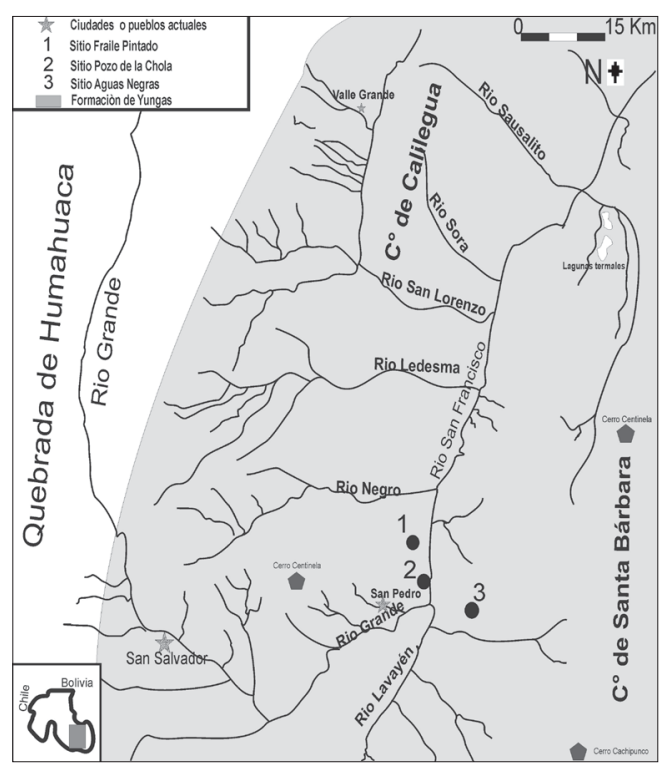

Figura 4: Mapa con la ubicación de los sitios donde fueron recuperados los fragmentos de cerámica analizados para ácidos grasos.

La extracción de los ácidos grasos de restos arqueológicos se realizó poniendo en contacto la parte a analizar con una solución de cloroformo: metanol-2:1. Se dejaron en reposo las muestras durante 12 horas y posteriormente, para mejorar la extracción, se sometió el conjunto a una etapa de sonicación de 30 minutos con ultrasonidos. La mezcla obtenida se colocó en un tubo Hach y se le agregaron $2 \mu \mathrm{L}$ de solución de $\mathrm{KOH}$ al $10 \%$ en metanol, agitando vigorosamente. El tubo fue herméticamente cerrado y calentado al baño María a $80^{\circ} \mathrm{C}$ durante 45 minutos. Se extrajo el material insaponificable con éter de petróleo, el cual fue desechado, y el resto se trató con $\mathrm{HCl}$ concentrado para producir la liberación de los ácidos grasos, los cuales fueron extraídos con éter de petróleo. El extracto etéreo se evaporó a sequedad en atmósfera de nitrógeno. Se agregó $1,5 \mu \mathrm{L}$ de $\mathrm{BF}_{3}$ al $10 \%$ en metanol, calentando durante $30 \mathrm{~min}$ a $80^{\mathrm{a}} \mathrm{C}$. El material derivado se pasó a un vial donde se evaporó a sequedad en atmósfera de $\mathrm{N}_{2}$ y se retomó con $1 \mu \mathrm{L}$ de hexano para luego ser cromatografiado. Se trabajó en un equipo Agilent Technologies 6890 acoplado a un espectrómetro de masas HP $5972^{\mathrm{a}} \mathrm{A}$, con inyección automática. Como estándar de comparación se utilizó el Estándar FAME MIX Supelco ${ }^{\mathrm{TM}} 37$ que posee 37 ácidos grasos metilados.

De las 21 muestras cromatografiadas, sólo 13 de ellas arrojaron resultados parciales (Cuadro 1). En los resultados obtenidos se observa que, en líneas generales, han sido identificados los ácidos grasos más frecuentes en la mayor parte de vegetales y animales, a saber: ácido mirístico, saturado de 14 átomos de carbono (C14:0), esteárico (C16:0), palmítico (C18:0), oleico, de 18 átomos de carbono con una insaturación (C18:1), linoleico (C18:2), linolénico (C18:3), araquídico (20:0) y behénico (22:0). Cuatro de las muestras fueron particularmente informativas. Dos de ellas (muestra 6 y 8 , Cuadro 1), correspondientes a fragmentos de cuerpos de botellas (una gris incisa y un fragmento bicolor pulido), presentaron perfiles que, de acuerdo con el estándar de comparación, podrían corresponder a la presencia de algarrobo. Para llegar a esta interpretación, se midieron las concentraciones relativas de los ácidos grasos de vainas de algarrobo de la región (Cuadro 2, Figura 5) y se buscaron antecedentes publicados para mediciones de la misma especie en otras regiones. En la variedad de algarrobo analizada en esta oportunidad, no aparece el ácido C14:0 (ácido mirístico), y el C18:3 ( $\gamma$-linolénico) tiene una importante abundancia relativa (Cuadro 2). Un trabajo realizado sobre vainas de Prosopis ruscifolia (Freyre et al. 2003) arrojó 
Cuadro 1: Ácidos grasos por muestra cerámica

\begin{tabular}{|c|c|c|c|c|c|c|c|c|c|c|c|c|c|}
\hline \multirow[b]{2}{*}{ 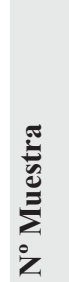 } & \multirow[b]{2}{*}{ 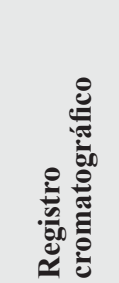 } & \multirow[b]{2}{*}{ 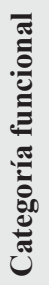 } & \multirow[b]{2}{*}{ 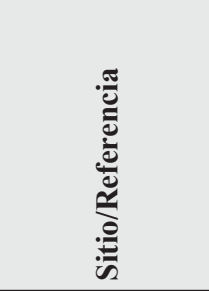 } & \multirow[b]{2}{*}{ 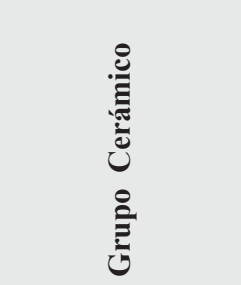 } & \multicolumn{9}{|c|}{ Ácidos grasos } \\
\hline & & & & & 窇 & 莺 & 赵 & $\frac{\frac{e}{0}}{0}$ & 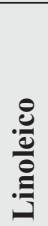 & 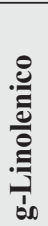 & 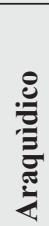 & 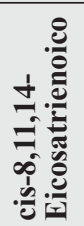 & 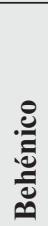 \\
\hline 1 & $10-1775$ & B & $\begin{array}{l}\text { PdCh UP Aa2 } \\
\text { Nivel VI }\end{array}$ & Gris pulido inciso & $X$ & $X$ & $X$ & - & - & - & - & $\mathrm{X}$ & $X$ \\
\hline 2 & $10-1778$ & $\mathrm{E}$ & AN F15/C11 & Gris pulido Inciso & - & $\mathrm{T}$ & - & - & - & - & . & - & - \\
\hline 3 & $10-1779$ & $?$ & $\begin{array}{l}\text { PdCH-UPA2 } \\
\text { NI } 69\end{array}$ & $\begin{array}{l}\text { Pintado bicolor } \\
\text { R/A }\end{array}$ & - & - & $\mathrm{T}$ & - & - & - & - & - & - \\
\hline 4 & $10-1782$ & $\mathrm{O}$ & $\begin{array}{l}\text { AN F4/C7 } \\
-1093\end{array}$ & Naranja alisado & - & $\mathrm{T}$ & - & - & - & - & - & - & - \\
\hline 5 & $10-1782$ & $\mathrm{O}$ & AN F9/C7 106 & Naranja alisado & - & - & $\mathrm{T}$ & - & - & - & - & - & - \\
\hline 6 & $10-1915$ & $\mathrm{~B}$ & AN F:13 C:9 & Gris alisada incisa & - & $\mathrm{X}$ & $\mathrm{X}$ & $\mathrm{X}$ & $\mathrm{X}$ & - & - & - & - \\
\hline 7 & $10-1916$ & V & $\begin{array}{l}\text { PdCh UP } 16 \\
\text { NII }\end{array}$ & $\begin{array}{l}\text { Oxidante naranja } \\
\text { incisa }\end{array}$ & - & $\mathrm{X}$ & $\mathrm{X}$ & $\mathrm{X}$ & $\mathrm{X}$ & - & - & - & - \\
\hline 8 & $10-1917$ & B & $\begin{array}{l}\text { PdCH UPA } 3,7 \text {, } \\
8,9,10,11,12\end{array}$ & $\begin{array}{l}\text { Pintada bicolor } \\
\text { R/A }\end{array}$ & - & $\mathrm{X}$ & $\mathrm{X}$ & $\mathrm{X}$ & $\mathrm{X}$ & - & - & - & - \\
\hline 9 & $10-1918$ & $\mathrm{~V}$ & $\mathrm{PdCh}$ & Alisada natural & - & $\mathrm{T}$ & - & - & - & - & - & - & - \\
\hline 10 & $10-1919$ & $\mathrm{~V}$ & FP & Naranja alisado & - & $\mathrm{T}$ & - & - & - & - & - & - & - \\
\hline 11 & $10-1921$ & $\mathrm{~B}$ & $\begin{array}{l}\mathrm{PdCh} \mathrm{C} / 1 \mathrm{~A} \\
\text { Nivel IV }\end{array}$ & Gris pulido inciso & - & $\mathrm{T}$ & $\mathrm{T}$ & - & - & - & - & - & - \\
\hline 12 & $10-1925$ & B & PdCh CM, N1 & $\begin{array}{l}\text { Pintada bicolor } \\
\mathrm{R} / \mathrm{N}\end{array}$ & - & $\mathrm{T}$ & - & - & - & - & - & - & - \\
\hline 13 & $10-1929$ & V & $\begin{array}{l}\mathrm{PdCH} \text { Sondeo } \\
2 \mathrm{C} / \mathrm{M} \text { relleno }\end{array}$ & Naranja alisado & - & $\mathrm{T}$ & $\mathrm{T}$ & - & - & - & - & - & - \\
\hline
\end{tabular}

Referencias: AN (Aguas Negras); PdCH (Pozo de la Chola); FP (Fraile Pintado). Categoría funcional: B (botella); V (vasija); O (olla) y E (escudilla); R/A (Rojo sobre Ante) y R/N (Rojo sobre Naranja); X (detectado); T (trazas).

Cuadro 2: Cantidad de ácidos grasos detectados en vainas de algarrobo actuales

\begin{tabular}{|c|c|c|c|c|c|c|c|c|c|c|}
\hline \multirow[b]{2}{*}{ 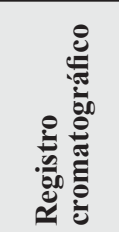 } & \multirow[b]{2}{*}{ 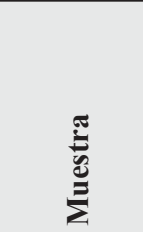 } & \multirow[b]{2}{*}{  } & \multicolumn{8}{|c|}{ Ácidos grasos } \\
\hline & & & 总总 & 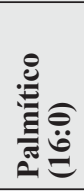 & 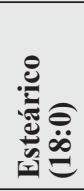 & 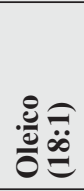 & 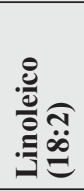 & 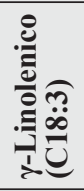 & 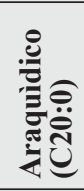 & 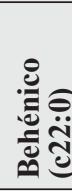 \\
\hline $10-1930$ & $\begin{array}{l}\text { Vaina de } \\
\text { algarrobo }\end{array}$ & $\begin{array}{l}\text { Prosopis chilensis } \\
\text { (NOA) }\end{array}$ & - & 36,4 & 4,5 & 20,6 & 25,6 & 7,6 & 1,5 & 0,7 \\
\hline $\begin{array}{l}\text { Freyre et } \\
\text { al. } 2003\end{array}$ & $\begin{array}{l}\text { Vaina de } \\
\text { algarrobo }\end{array}$ & $\begin{array}{l}\text { Prosopis ruscifolia } \\
\text { (México) }\end{array}$ & 1,3 & 9,5 & 4,7 & 28,5 & 49,8 & 1,7 & 3,4 & - \\
\hline
\end{tabular}

resultados dispares a los nuestros, a pesar de que los autores expresan que los resultados publicados para especies de Prosopis de Argentina eran coincidentes con los de su trabajo. En los resultados dados a conocer por Freyre y colaboradores aparece el mirístico con una pequeña concentración y el linolénico con una concentración muy inferior a la detectada en las vainas analizadas en nuestro trabajo. Es entonces posible 


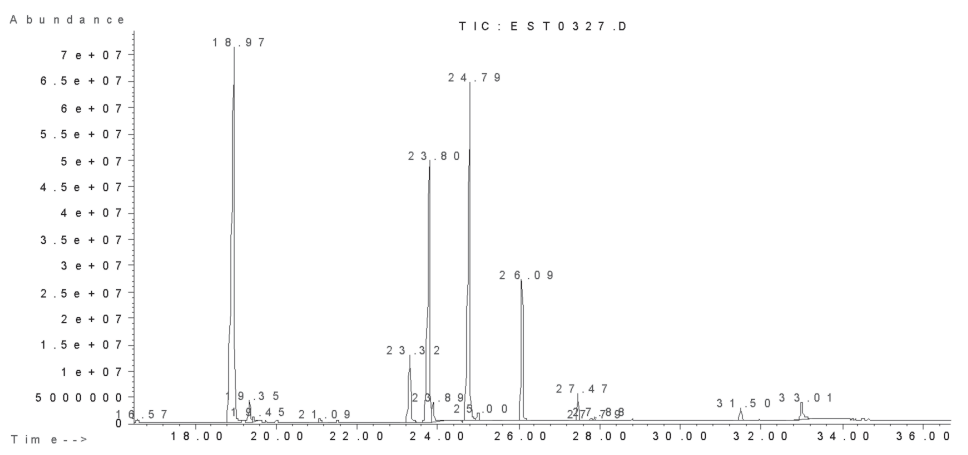

Figura 5: Perfil cromatográfico de vainas de Prosopis chilensis.

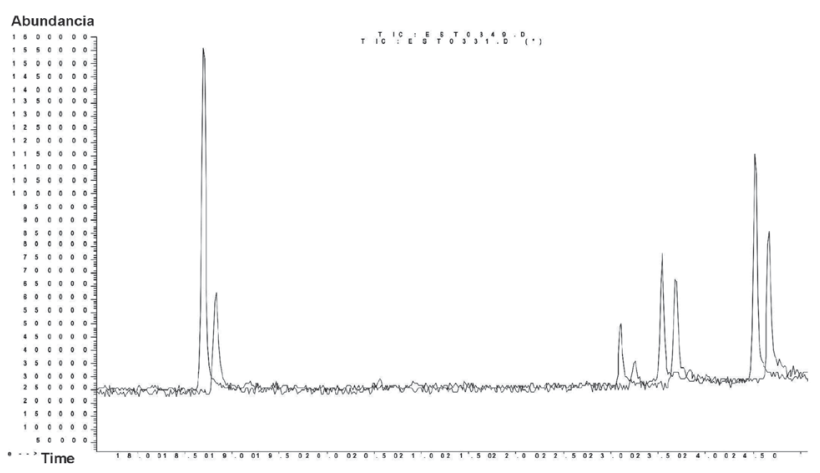

Figura 6: Perfil cromatográfico de la muestra $\mathrm{N}^{\circ} 7$ (Cuadro 1). Los picos más pequeños corresponden al perfil obtenido de la superficie interna del tiesto cerámico. Los picos más altos superpuestos corresponden al perfil obtenido de una variedad de maíz moderna (variedad harinoso).

que la variedad utilizada en contextos arqueológicos haya tenido valores más similares a los registrados para la variedad ruscifolia, lo que podría explicar la ausencia del ácido linolénico en las piezas arqueológicas.

Por último, la muestra 7 (Cuadro 1), correspondiente al fragmento de una vasija tipo cántaro, arrojó valores más cercanos a los perfiles de comparación del maíz (Figura 6). Estos resultados sugieren que en dos casos como mínimo el algarrobo estuvo presente en estos recipientes y que, en un tercero, posiblemente hubo maíz. Dado que en los dos primeros se trata de botellas, es posible que hayan contenido aloja y chicha. Ambas son cervezas nativas obtenidas a partir de la molienda y fermentación de las vainas del árbol de algarrobo (Prosopis sp.) (Figura 7), en el caso de la aloja, y del maíz (Zea mays) en el de la chicha. En el pasado cercano y aún en el presente ambas bebidas, de elevado contenido alcohólico, son populares en algunas regiones del noroeste de Argentina, donde se consumen en ocasiones festivas y siguen siendo elaboradas de manera tradicional. En la prehistoria del NOA el uso del algarrobo ha sido ampliamente documentado en contextos de diferente tipo (Giovanetti et al. 2008), mientras que la chicha debe haber tenido una importancia muy grande para diferentes poblaciones del noroeste según se desprende de la documentación etnohistórica (Cremonte et al. 2009). Si la muestra 7 pudo contener bebida a base de maíz, dado que se trata de un tipo de contenedor más apto para la fermentación o almacenamiento, conociendo los procesos de preparación de ambas bebidas es posible sugerir que los distintos contenedores se usaron en diferentes fases de elaboración y consumo. La va- 


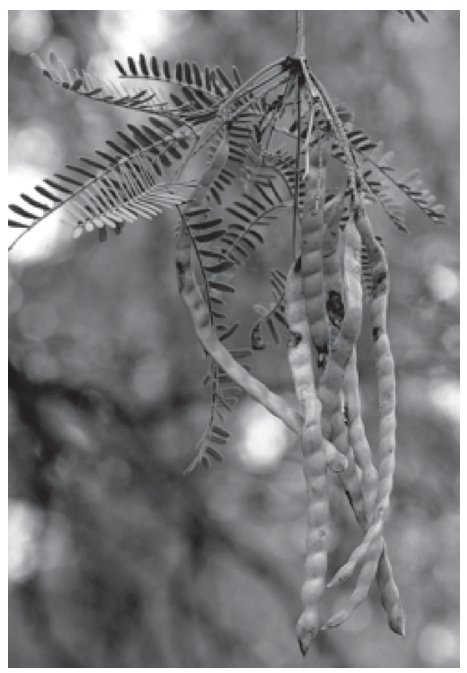

Figura 7: Vainas de

Prosopis actuales.

Figura 8: Fragmentos de cerámica analizados correspondientes a diferentes clases de contenedores: A) vasija tipo cántaro; B) botellas; y C) pieza cerrada pintada en rojo sobre ante, posiblemente otra botella.

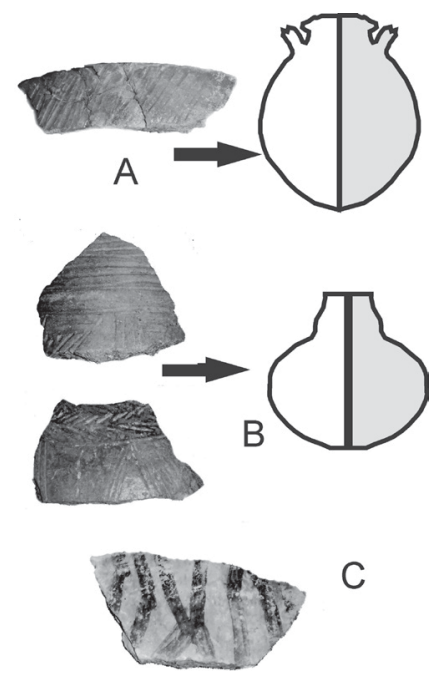

sija oxidante pudo ser empleada para la fermentación o el almacenamiento, mientras que las botellas, dada su función para verter y el despliegue visual de la decoración en el caso del fragmento correspondiente a una pieza pulida y pintada, pudieron ser empleadas para servir la bebida (Figura 8).

Por otro lado, una última muestra (número 1, Cuadro 1) correspondiente a una pieza cerrada, posiblemente una botella, gris incisa presentó un ácido graso muy infrecuente debido a que su estructura química es más frágil y sensible a las transformaciones, por lo que la posibilidad de su preservación es altamente infrecuente. Se trata del ácido cis-8,11,14-eicosatrienoico, que pertenece a la familia de los ácidos poliinsaturados de cadena larga de la serie omega 6. Este ácido graso sólo se encuentra en estado natural en peces y mamíferos marinos, así como algunos peces de río. Ha sido registrado en muestras modernas de peces de agua dulce pertenecientes a la cuenca del Paraná-Plata, particularmente dorado (Salminus maxillosus) y patí ( $\mathrm{Lu}$ ciopimelodus pati). Ambas especies se encuentran hasta el presente en la cuenca del río San Francisco. Dado el hallazgo de restos de ictiofauna en sitios arqueológicos de la región, y en particular en el sitio de donde proviene esta muestra, en la que se han identificado vértebras quemadas de Salminus maxillosus (Killian com. pers.), la presencia de este ácido graso reforzaría la interpretación del consumo o procesamiento de especies nativas de peces.

\subsection{Análisis de fitolitos y almidones}

Los almidones y fitolitos pertenecen a la categoría de microrrestos vegetales. Debido a sus características particulares de supervivencia y a su valor taxonómico, desde hace un par de décadas (Pearsall 1989) los microrrestos han comenzado a ser utilizados en el ámbito de la arqueología, como indicadores o elementos diagnósticos de la presencia de plantas en lugares en donde los procesos de descomposición de la materia orgánica no han permitido la supervivencia de macrorrestos vegetales (Piperno 
Cuadro 3: Abundancia relativa de microrrestos (fitolitos y almidones) por muestra

\begin{tabular}{|c|c|c|c|c|}
\hline Muestra & & & & \\
\hline \multirow{5}{*}{2219} & \multirow{2}{*}{$\mathrm{A}+\mathrm{C}$} & Fitolitos & Muy frecuente & Fitolitos \\
\hline & & Almidón & Raro & Almidón \\
\hline & $\mathrm{B}$ & & Frecuente & Fitolitos \\
\hline & $\mathrm{D}$ & & Ausente & \\
\hline & $\mathrm{E}$ & & Escaso & Fitolitos \\
\hline \multirow{2}{*}{2220} & \multirow{2}{*}{\multicolumn{2}{|c|}{$\begin{array}{l}53-250 \text { mic. } \\
5-53 \text { mic. }\end{array}$}} & Escaso & Espículas \\
\hline & & & Escaso & Espículas y escasos fitolitos \\
\hline \multirow{5}{*}{2221} & \multirow{2}{*}{$\mathrm{A}+\mathrm{C}$} & Fitolitos & Escaso & Fitolitos \\
\hline & & Almidón & Escaso & Almidón \\
\hline & B & & Frecuente & Fitolitos \\
\hline & $\mathrm{D}$ & & Ausente & \\
\hline & $\mathrm{E}$ & & Escaso & Fitolitos \\
\hline \multirow{2}{*}{2222} & \multirow{2}{*}{\multicolumn{2}{|c|}{$\begin{array}{l}53-250 \text { mic. } \\
5-53 \text { mic. }\end{array}$}} & Escaso & Espículas \\
\hline & & & Escaso & Espículas y escasos fitolitos \\
\hline \multirow{5}{*}{2223} & \multirow{2}{*}{$\mathrm{A}+\mathrm{C}$} & Fitolitos & Frecuente & Fitolitos \\
\hline & & Almidón & Escaso & Almidón \\
\hline & $\mathrm{B}$ & & Frecuente & Fitolitos \\
\hline & $\mathrm{D}$ & & Ausente & \\
\hline & $\mathrm{E}$ & & Escaso & Fitolitos \\
\hline \multirow{5}{*}{2224} & \multirow{2}{*}{$\mathrm{A}+\mathrm{C}$} & Fitolitos & Escaso & Fitolitos y diatomeas \\
\hline & & Almidón & Muy frecuente & Almidón \\
\hline & $\mathrm{B}$ & & Frecuente & Fitolitos \\
\hline & $\mathrm{D}$ & & Ausente & \\
\hline & $\mathrm{E}$ & & Escaso & Fitolitos \\
\hline \multirow{2}{*}{2225} & \multirow{2}{*}{\multicolumn{2}{|c|}{$\begin{array}{l}53-250 \text { mic. } \\
5-53 \text { mic. }\end{array}$}} & Ausente & \\
\hline & & & Frecuente & \\
\hline \multirow{2}{*}{2226} & \multirow{2}{*}{\multicolumn{2}{|c|}{$53-250$ mic. }} & Frecuente & \\
\hline & & & Escaso & Espículas y fitolitos \\
\hline
\end{tabular}

Referencias: $\mathrm{A}+\mathrm{C}$ (cara y superficie interna); $\mathrm{B}+\mathrm{D}$ (cara y superficie externa) y E (matriz).

y Pearsall 1998). Cuando una planta muere, sus partes perecederas se descomponen, pero los microrrestos pueden ser liberados y acumulados en un lugar próximo, con altas expectativas de conservación, como componentes estables en suelos y artefactos, aun en sitios a cielo abierto (Babot 2007).

Para la primera experiencia de este tipo de estudios en materiales de la región pedemontana de Jujuy se llevó a cabo una aproximación prospectiva, con efecto de probar la factibilidad de preservación de microrrestos en tiestos y sedimentos. Por ese motivo la identificación no tuvo parámetros porcentuales, siendo su finalidad establecer pautas de presencia (Zucol y Colobig 2010). La metodología utilizada para la concentración de los microrrestos silíceos se basó en las pautas metodológicas convencionales utilizadas para la concentración de biominerales en materiales clásticos (Bonomo et al. 2008, citado en Zucol y Colobig 2010); para la concentración de los granos de almidón se adaptó la metodología propuesta por Pearsall y colaboradores y por Horrocks (Zucol y Colobig 2010). Las observaciones microscópicas fueron realizadas en un microscopio Nikon Eclipse E200.

Las muestras utilizadas provienen del sitio Pozo de la Chola $(\mathrm{PdCH})$ y corresponden a 4 tiestos de cerámica y 4 muestras del sedimento de los pisos de ocupación del 
Cuadro 4: Tipo de alteraciones y abundancia en los microrrestos (fitolitos y almidones) por muestra cerámica

\begin{tabular}{|c|c|c|c|c|c|}
\hline $\begin{array}{c}\mathrm{N}^{\circ} \text { de } \\
\text { muestra }\end{array}$ & $\begin{array}{l}\text { Tipo } \\
\text { cerámico }\end{array}$ & $\begin{array}{l}\text { Abundan- } \\
\text { cia de mi- } \\
\text { crorrestos }\end{array}$ & $\begin{array}{l}\text { Restos } \\
\text { silíceos }\end{array}$ & $\begin{array}{c}\text { Tipo de } \\
\text { alteración en } \\
\text { almidones }\end{array}$ & $\begin{array}{l}\text { Morfología de } \\
\text { almidones }\end{array}$ \\
\hline 2219 & $\begin{array}{l}\text { Oxidante inciso } \\
\text { externo }\end{array}$ & escaso & $\begin{array}{l}\text { elementos grami- } \\
\text { noides halterifor- } \\
\text { mes (panicoideas) }\end{array}$ & poco alterados & $\begin{array}{l}\text { esféricos a ovales y } \\
\text { de contorno cuadrado }\end{array}$ \\
\hline 2221 & Oxidante liso & escaso & $\begin{array}{l}\text { elementos de con- } \\
\text { torno ondulados o } \\
\text { festoneado simila- } \\
\text { res a las cubiertas } \\
\text { de protección de } \\
\text { graminoides (espi- } \\
\text { guillas) }\end{array}$ & $\begin{array}{l}\text { masa almido- } \\
\text { nosa aglutina- } \\
\text { da (modifica- } \\
\text { ción química) }\end{array}$ & $\begin{array}{l}\text { esféricos a ova- } \\
\text { les (con cruz de } \\
\text { extinción simétrica y } \\
\text { asimétrica). Escasos } \\
\text { de contorno cuadrado }\end{array}$ \\
\hline 2223 & Oxidante liso & moderados & $\begin{array}{l}\text { elementos prismá- } \\
\text { ticos elongados } \\
\text { y poliédricos y } \\
\text { material ambarino }\end{array}$ & $\begin{array}{l}\text { masa almido- } \\
\text { nosa aglutina- } \\
\text { da (modificia- } \\
\text { ción química) }\end{array}$ & $\begin{array}{l}\text { esféricos a ovales, re- } \\
\text { niformes y en forma } \\
\text { de lágrima }\end{array}$ \\
\hline 2224 & Oxidante liso & abundantes & Diatomeas & $\begin{array}{l}\text { medianamen- } \\
\text { te alterados } \\
\text { (modificación } \\
\text { mecánica) }\end{array}$ & $\begin{array}{l}\text { esféricos a ovales, } \\
\text { lisos y concéntricos; } \\
\text { escasos de contorno } \\
\text { cuadrado }\end{array}$ \\
\hline
\end{tabular}

sitio. Los fragmentos de cerámica corresponden a vasijas cerradas de cocción oxidante, uno de ellos con la superficie externa estriada y con incisiones lineales poco profundas (muestra $\mathrm{n}^{\circ} 2221$ ). Este fragmento se corresponde con el mismo tipo cerámico al que pertenece el fragmento 10-1916 (muestra 7, Cuadro 1) sometido a análisis de ácidos grasos. Las muestras de tiestos fueron subdivididas en 5 submuestras correspondientes a sendas superficies de ambas caras (externa e interna) y a la matriz de los fragmentos. La mejor preservación de microrrestos fue observada en las submuestras de las caras internas, mientras que las superficies externas presentaron muy poco o ningún vestigio. Las mayores variaciones observadas se observan tanto en lo referente a microrrestos silíceos como a la presencia de sustancias almidonosas (Cuadro 3).

La muestra 2224 es la que presentó la mayor abundancia de material almidonoso y en menor medida la 2219, siendo escasa su presencia en otras dos muestras (Zucol y Colobig 2010). En lo que hace al estado de conservación, la muestra 2224 presentó el material en mejor estado, mientras que las muestras 2221 y 2223 presentaron una elevada cantidad de sustancia almidonosa en masa, condición que en menor medida también se observó en la muestra 2219 (Cuadro 4)

En cuanto a la presencia de microrrestos silíceos, la muestra 2219 presentó en forma diferencial elementos graminoides halteriformes que se asignan a las panicoideas $^{2}$. La submuestra 2221 se caracterizó por la presencia de elementos silicificados de contorno ondulado o festoneado, articulados de forma similar a las cubiertas de protección de muchos granos graminoides (Zucol y Colobig 2010).

2 Las panicoideas corresponden a una subfamilia de las Poaceae o gramíneas, dentro de la cual se encuentran las Maideas. La especie doméstica se corresponde con el maíz 


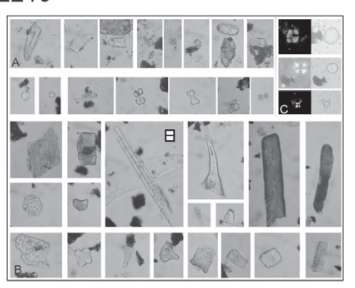

2221

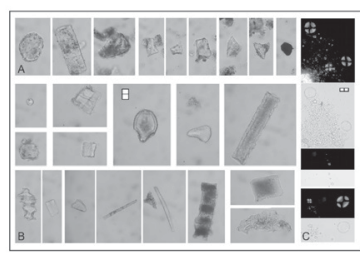

2223

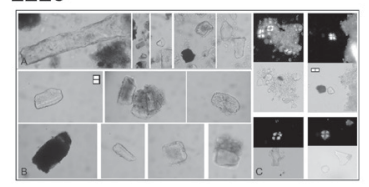

2224



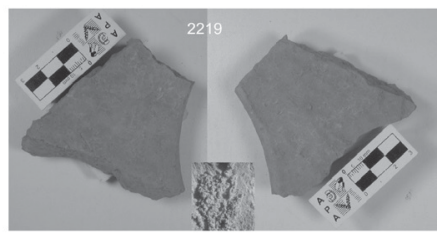

Figura 9: Fragmentos de cerámica del sitio Pozo de la Chola analizados para fitolitos y almidones. Las fotos de la derecha corresponden a cada uno de los tiestos y las de la izquierda a los diferentes tipos de microrrestos encontrados en cada muestra bajo luz normal y luz polarizada.
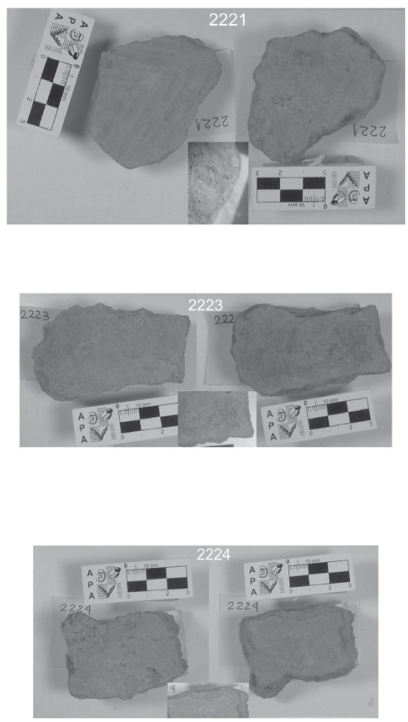

Entre los almidones identificados figuran los de contorno cuadrado, que en algunas muestras presentaron fisuras y se ajustan a las descritas para especies de Prosopis (P.chilensis y P. flexuosa) (Giovannetti et al. 2008) ${ }^{3}$ (Figura 9). En lo que respecta a los granos de almidón, tanto los esféricos como los redondeados están vinculados a las maideas (Zucol y Colobig 2010). Esto coincide con la presencia diferencial de microrrestos panicoides y fitolitos articulados originados en espiguillas de gramíneas de las muestras 2221 y 2219 (Figura 9). Por su parte algunos granos de forma piriforme son asociados con especies de Phaseolus sp. (Babot et al. 2007) conjuntamente con otros granos de almidón pequeños y aglomerados. Estos últimos han sido descritos como pertenecientes a pseudocereales de las familias de las amarantáceas y las quenopodiáceas ${ }^{4}$. Por último los granos de almidón grandes más o menos reniformes

3 El género Prosopis pertenece a la subfamilia Mimosaceae y crece en ambientes semiáridos a áridos o con estación seca. Este género tiene una alta variabilidad morfológica y ocupa territorios de gran diversidad climática y edáfica. En Argentina existen 4 especies P. chilensis, P. flexuosa, P. alba y P. nigra. En la antigüedad fue ampliamente explotado por las poblaciones precolombinas del noroeste de Argentina siendo considerado un recurso de gran importancia.

4 Las quenopodáceas son plantas herbáceas correspondientes a una subfamilia de las amarantáceas. Agrupa a más de 1400 especies. El género Chenopodium contiene muchas plantas de importancia para la alimenta- 
Cuadro 5: Características y abundancia de micro-restos silíceos por muestra sedimentaria (sitio Pozo de la Chola)

\begin{tabular}{|c|c|c|c|c|}
\hline $\begin{array}{c}\mathrm{N}^{0} \text { de } \\
\text { muestra }\end{array}$ & $\begin{array}{c}\text { Tipo de } \\
\text { sedimento }\end{array}$ & $\begin{array}{c}\text { Nivel de } \\
\text { procedencia }\end{array}$ & Restos silíceos & Abundancia \\
\hline 2220 & limoarcilloso & Nivel III & $\begin{array}{l}\text { Espongiarios. Fitolitos poliédricos y } \\
\text { escasos prismáticos elongados }\end{array}$ & abundantes \\
\hline 2222 & limoarcilloso & Nivel III & $\begin{array}{l}\text { Espículas de espongiarios políedricos y } \\
\text { prismáticos. Material clástico ambarino }\end{array}$ & escasos \\
\hline 2225 & limoarcilloso & $\begin{array}{l}\text { Nivel IV lente } \\
\text { fogonosa }\end{array}$ & $\begin{array}{l}\text { Macroescleras de espongiarios y fitoli- } \\
\text { tos prismáticos }\end{array}$ & escasos \\
\hline 2226 & limoarcilloso & Nivel IV & $\begin{array}{l}\text { Biominerales asociados a fragmentos } \\
\text { carbonosos }\end{array}$ & escasos \\
\hline
\end{tabular}

podrían estar vinculados a especies de solanáceas del tipo de la papa (Solanum sp.) (Zucol y Colobig 2010). En relación con los tipos de alteraciones observadas en los granos, existen variaciones que tienen que ver con una posible manipulación mecánica (molido) o por acción de la cocción (hervido).

Por su parte, las muestras sedimentarias fueron positivas para fitolitos pudiéndose probar que la matriz sedimentaria en donde fueron desenterrados los tiestos cerámicos tuvo poca influencia en la presencia de microrrestos en los fragmentos arqueológicos (Cuadro 5). La presencia de fitolitos en estas muestras fue muy baja siendo las espículas de espongiarios, de los tipos macroscleras lisas, los más representados. El análisis comparado indica, en este lugar en particular, una vinculación con un ambiente fluvial dominado por un ambiente enérgico. Esto es del todo coherente con el emplazamiento del sitio arqueológico, que se encuentra actualmente ubicado sobre una de las terrazas del río San Francisco (Figura 10).

El resultado de los análisis de fitolitos proporciona información suplementaria acerca de la manufactura de las vasijas, ya que existe un elevado grado de homogeneidad en la composición de las pastas, habiéndose detectado fitolitos de palmeras en su contenido, lo que nos informa acerca de las características del ambiente en donde fueron elaboradas y sugiriendo una misma fuente de aprovisionamiento de arcilla.

\section{Discusión}

Durante más de un siglo, desde que se realizaron los primeros descubrimientos arqueológicos en la región, se asumió que las poblaciones tempranas que ocuparon la cuenca del valle de San Francisco fueron poblaciones agricultoras. Sin embargo, no se contaba con pruebas directas acerca de la presencia de plantas domésticas, y mucho menos aún, sobre la importancia que estas pudieron tener en la economía de estas poblaciones. Debido a las condiciones ambientales y a las características pedológicas de los suelos, en más de cien años de excavaciones discontinuas se recuperaron esca-

ción ya que son clasificadas como pseudocereales. En el NOA la variedad Chenopodium quinoa fue un recurso de gran importancia para los pueblos andinos. En el caso de las quenopodáceas identificadas en esta oportunidad, podrían corresponder a variedades domésticas por el tamaño de los almidones, sin embargo resta aún determinar a cual especie corresponde ya que no es el ambiente apto para el crecimiento de la variedad Quinoa. 


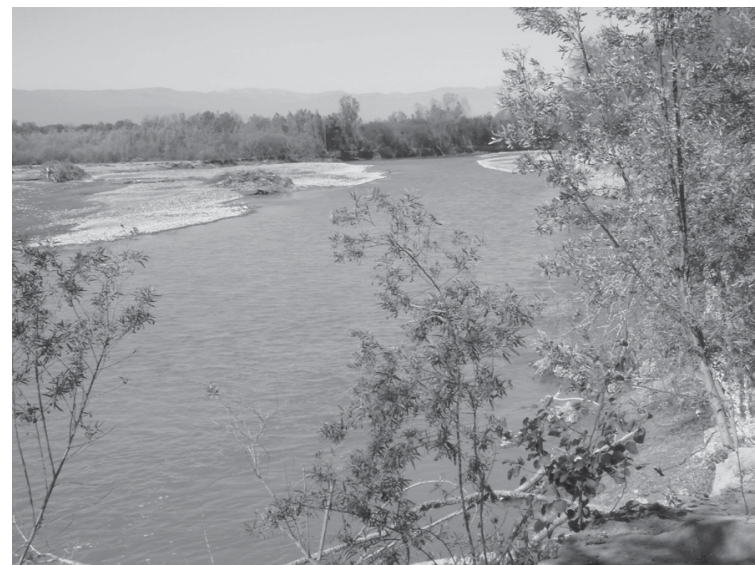

Figura 10: Vista de un brazo del río San Francisco desde el sitio arqueológico Pozo de la Chola (fotografía tomada en invierno, mes de julio). Al fondo de observa la serranía de Santa Bárbara, cordón de las Sierras Subandinas.

sos restos macrobotánicos, por no decir prácticamente ninguno. Sobre la base de una supuesta economía principalmente agrícola, estos grupos se consideraron sociedades formativas en la escala de desarrollo cultural del noroeste de Argentina.

Nuevas tecnologías y metodologías, desarrolladas especialmente en las últimas dos décadas, han provisto a la arqueología de las herramientas necesarias para detectar restos materiales imposibles de recuperar con los métodos tradicionales. Entre ellas son especialmente valiosas las derivadas de la botánica y la química, que han permitido la recuperación de diversos tipos de microrrestos a pesar de las condiciones deficientes de preservación en climas desfavorables.

Los resultados presentados aquí nos permiten sostener que las plantas domésticas formaron parte de los recursos consumidos por estas poblaciones. Entre las especies identificadas, podemos mencionar aquellas plantas que han sido las más importantes en la economía de la mayoría de las poblaciones del noroeste de Argentina: el poroto y el maíz. Llamativamente, aún no se ha identificado zapallo (Curcubita sp.) a pesar de que la región presenta las condiciones óptimas para su crecimiento ${ }^{5}$. Otras especies identificadas corresponden a alguna variedad de papa y a quenopodáceas. El algarrobo muestra una presencia significativa, indicando la relevancia de este recurso silvestre en el pasado. Tanto el maíz como el algarrobo pueden haber tenido una importancia muy grande asociada con la fermentación y elaboración de bebidas, restando aún comprobar la importancia de ambos como alimentos en la economía doméstica. La identificación de ácidos grasos de pescado refuerza, por otra parte, los datos aportados por el registro ictioarqueológico, indicando la explotación de este recurso en el pasado.

Resta aún evaluar la importancia relativa de plantas y animales silvestres frente a los domesticados en la economía de estas poblaciones. Medir el grado de impacto en la dieta de las diferentes clases de recursos (animales frente a vegetales, plantas de diferente patrón fotosintético, etc.) nos permitirá discutir en el futuro otros aspectos de la organización socio-productiva de estos grupos, así como su adscrito estatus «formativo».

5 El ají (Capsicum sp.) y el zapallo (Curcubita sp) presentan muy escasa cantidad de almidones, por lo que no es esperable encontrarlos a partir de este tipo de estudios (Giovannetti com. pers.). 
Agradecimientos: Este trabajo forma parte del proyecto PIP de CONICET 11420090100180 dirigido por la primera de las autoras, del proyecto PICTO-UNJu 08-00131 y del proyecto SECTERUNJu C/0102. Agradecemos a la Lic. Agustina Scaro la traducción al inglés del resumen en español.

\section{Referencias bibliograficas}

BАвот, María del Pilar

2007 «Almidones y fitolitos: desentrañando el papel funcional de los artefactos de molienda arqueológicos», en Arqueología argentina en los inicios de un nuevo siglo. Publicación del XIV Congreso Nacional de Arqueología Argentina, Tomo III, Fernando Oliva, Nélida de Grandis y Jorge Rodríguez, comps., pp. 665-673. Rosario: Universidad Nacional de Rosario.

BАвот, María del Pilar, Nurit Oliszewski y Alfredo Grau

2007 «Análisis de caracteres macroscópicos y microscópicos de Phaseolus vulgaris (fabaceae, faboideae) silvestres y cultivados del noroeste argentino: una aplicación en Arqueobotánica». Darwiniana 45 (2): 149-162.

BOMAN, Eric

1991 Antigüedades de la región andina de la República Argentina y del desierto de Atacama [1908]. San Salvador de Jujuy: Universidad Nacional de Jujuy.

Cremonte, María Beatriz, Clarisa Otero y María Soledad Gheggi

2009 «Reflexiones sobre el consumo de chicha en épocas prehispánicas a partir de un registro actual en Perchel (Dto. Tilcara, Jujuy)». Relaciones de la Sociedad Argentina de Antropología 34: 75-103.

DOUGHerTy, Bernardo

1975 Nuevos aportes para el conocimiento del Complejo Arqueológico San Francisco (sector septentrional de la región de las selvas occidentales argentinas, subárea del noroeste argentino). Tesis doctoral inédita. Universidad Nacional de La Plata.

Dougherty, Bernardo, Carlos de Feo y Ana Fernández

2003 «El yacimiento arqueológico El Fuerte (Dpto. Santa Bárbara, Pcia. de Jujuy). Su ubicación en el complejo arqueológico San Francisco», en La mitad verde del mundo andino. Investigaciones arqueológicas en la vertiente oriental de los Andes y las tierras bajas de Bolivia y Argentina, Gabriela Oritz y Beatriz Ventura, eds., pp. 73-98. San Salvador de Jujuy: Universidad Nacional Jujuy.

DudD, Stephanie y Richard P. Evershed

1999 «Evidence for Varying Patterns of Exploitation of Animal Products in Different Prehistoric Pottery Traditions Based on Lipids Preserved in Surface and Absorbed Residues». Journal of Archaeological Science 26: 1473-1482.

ECHENIQUe, Mónica y Jorge Kulemeyer

2003 «La excavación arqueológica de una 'mancha blanca', el sector M43C en el sitio Moralito, departamento San Pedro, provincia de Jujuy, República Argentina», en $L a$ mitad verde del mundo andino. Investigaciones arqueológicas en la vertiente oriental de los Andes y las tierras bajas de Bolivia y Argentina, Gabriela Oritz y Beatriz Ventura, eds., pp. 99-132. San Salvador de Jujuy: Universidad Nacional Jujuy.

EERKENS, Jelmer W.

2007 «Organic Residue Analysis and the Decomposition of Fatty Acids in Ancient Pots- 
herd», en Theory and Practice in Archaeological Residue Analysis, H. Barnard y J.W. Eerkens, eds., pp. 90-98. B.A.R International Series 1650. Oxford: Archaeopress.

FERNÁNDEZ Distel, Alicia

1994 «Noticia sobre el sitio arqueológico de Abra de los Morteros y otros lugares de valor prehistórico en la región de Santa Bárbara (Jujuy, Rep. Argentina)», en De costa a selva: producción e intercambio entre los pueblos agroalfareros de los Andes Centro-Sur, María E. Albeck, ed., pp. 255-300. Buenos Aires: Universidad de Buenos Aires.

Freyre, M., E. Astrada, C. Blasco, C. Baigorria, V. Rozycki y C. Bernardi

2003 «Valores nutricionales de frutos de vinal (Prosopis ruscifolia): consumo humano y animal». Ciencia y Tecnología Alimentaria 4 (1): 41-46.

Giovannetti, Marcos, Verónica Lema, Carlos Bartoli y Aylen Capparelli

2008 «Starch Grain Characterization of Prosopis chilensis (Mol.) Stuntz and P. flexuosa DC, and the Analysis of Their Archaeological Remains in Andean South America». Journal of Archaeological Science 35 (11): 2973-2985.

LAGUENS, Andres

1993 «Locational Structure of Archaeological Underground Storage Pits in Northwest Córdoba, Argentina». Revista do Museu de Arqueologia e Etnologia 3: 17-33. São Paulo.

LuPo, Liliana y Mónica EcHENIQUE

2001 «Reconstrucción arqueopalinológica de los distintos momentos de ocupación del Yacimiento Formativo Moralito, Jujuy, noroeste argentino». Ameghiniana, n especial: 125-130.

NORDENSKIÖLD, Erland

1993 Lugares precolombinos de asentamiento y entierro en la frontera sudoeste del Chaco [1903]. San Salvador de Jujuy: Universidad Nacional de Jujuy.

OrTIZ, Gabriela

2007 La evolución del uso del espacio en las tierras bajas jujeñas (subárea del río San Francisco). Tesis doctoral inédita. Universidad Nacional de Córdoba.

2011 «Pescadores, cazadores, recolectores pedemontanos? El caso de las sociedades de tradición San Francisco (prov. de Jujuy, noroeste de argentina)». CazadoresRecolectores del Cono Sur. Revista de Arqueología 4: 115-134.

Pearsall, Deborah

1989 Paleoethnobotany. A Handbook of Procedures. San Diego: Academic Press.

PIPERNo, Dolores y Deborah PeARsall

1998 The Origins of Agriculture in the Lowland Neotropics. San Diego: Academic Press.

SELDEs, Verónica y Gabriela OrTIZ

2009 «Avances en los estudios bioarqueólogicos de la región del río San Francisco, Jujuy, Argentina». Andes 20: 15-35.

Sмiтн, Bruce D.

2001 «Low-Level Food Production». Journal of Archaeological Research 9 (1): 1-43.

Zucol, Alejandro F. y María de los Milagros CoLoBiG

2010 Análisis de presencia de microrestos en fragmentos de cerámica de la localidad arqueológica Pozo de la Chola, Jujuy, Argentina. Informes del Laboratorio de Paleobotánica 19 (CICYTTP-Diamante). Informe inédito. 